\title{
Idiopathic Neuralgic Amyotrophy in Childhood
}

Author

Affiliation

\section{Kotsopoulos ${ }^{1,4}$, K. Faber ${ }^{1}$, J. Raaijmakers ${ }^{2}$, N. Van Alfen ${ }^{3}$, J. Nicolai ${ }^{1}$, V. van Kranen-Mastenbroek ${ }^{4}$}

${ }^{1}$ Department of Neurology, University Hospital Maastricht, Maastricht, The Netherlands

${ }^{2}$ Department of Pediatrics, University Hospital Maastricht, Maastricht, The Netherlands

${ }^{3}$ Departments of Clinical Neurophysiology, Radboud University Nijmegen Medical Centre, Nijmegen, The Netherlands

${ }^{4}$ Department of Clinical Neurophysiology, University Hospital Maastricht, Maastricht, The Netherlands
Key words

- Neuralgic amyotrophy

- bilateral plexus neuropathy

- osteomyelitis

\section{Abstract \\ $\nabla$}

In addition to the review by van Alfen et al., which has been published in 2000 , we would like to report a seven-week-old boy with bilateral

\section{Introduction}

$\nabla$

In the article by Alfen et al. [1] two children with idiopathic neuralgic amyotrophy are described and combined with a review of the literature. As the authors pointed out, this disorder is rare and, at publication in 2000 , there were no reports of bilateral plexus involvement in children. In addition to this review, we would like to report a seven-week-old boy with bilateral idiopathic brachial plexus neuropathy that showed no signs of osteomyelitis on repeated scintigraphy.

\section{Case Report}

At the age of seven weeks a male infant presented with a subacute loss of spontaneous movements in his upper arms. Since 5 days he showed irritability during daily care and there were mild feeding problems. His mother had noted temperatures up to $38^{\circ} \mathrm{C}$. The further medical and family history were unremarkable; specifically there was no history of trauma. Upon physical examination, he was found to have a bilateral flaccid paralysis of the deltoideus, biceps, and triceps muscles, and a normal biceps reflex on both arms.

$\mathrm{X}$-rays of the arms and cervical spine were normal. MRI scanning of the cervical spinal cord to rule out a cervical abscess or hematoma was performed on the day of admission and showed no abnormalities.

Concentric needle elecromyography showed positive sharp waves and fibrillations in the deltoid, biceps, triceps, and flexor carpi radialis idiopathic brachial plexus neuropathy that did not show any signs of osteomyelitis on repeated scintigraphy. To our knowledge this is the first child reported with bilateral plexus involvement.

muscles on the right, and in the biceps muscle on the left. The paravertebral muscles at the level of the cervical vertebrae $\mathrm{C} 5-\mathrm{C} 7$ as well as the right abductor pollicis brevis and the muscles of the legs were unaffected. The conduction velocities were normal and there were no signs of a neuromuscular transmission disorder due to botulism as investigated by repetitive nerve stimulation. Considering these findings we concluded to a bilateral upper brachial plexus lesion.

Routine blood studies showed an increased level of C-reactive protein (CRP) (172 mg/l; reference $2-9)$, thrombocytes $\left(583 \times 10^{9} / 1\right.$; reference 130 350 ), and slightly elevated gamma glutamyl transferase (GGTP) (256U/1; reference 0-200) with normal transaminases and bilirubin. Cerebrospinal fluid (CSF) cell count, protein, and glucose levels were normal. CSF virology and serology analyses for cytomegalovirus, EpsteinBarr virus, herpes simplex virus, varicella zoster virus, coxsackie virus, echovirus, adenovirus, enterovirus, and mycoplasma pneumoniae were all negative. The serum anti-streptodornase and anti-streptolysine levels were normal. All screening tests in faeces for enterovirus, adenovirus, rotavirus, poliovirus were negative as well as analyses in serum and in faeces for clostridium botulinum toxins. As in children under 8 weeks of age a concomitant osteomyelitis of the affected shoulder or arm always seems to be involved [1], a skeleton scintigraphy was performed twice without detecting any signs of an osteomyelitis. Because of the previously noted fever and the elevated CRP, the child was suspected of having 
an infection and was temporarily treated with amoxicillin/ clavulanic acid and gentamicin.

After exclusion of other causes, the diagnosis of idiopathic neuralgic amyotrophy was made and the child received prednisolone ( $1 \mathrm{mg} / \mathrm{kg} /$ day) intravenously during one week and a tapering scheme in the second week. On this treatment there was a marked improvement of irritability possibly due to pain reduction and of the motor function of the upper arms. At follow-up one month later the motor function of the upper arms had normalized except of a slight weakness of the shoulder muscles on vertical suspension.

\section{Discussion}

\section{$\nabla$}

The clinical features of our patient are of particular interest since they reveal that the clinical spectrum of childhood neuralgic amyotrophy might be broader than previously reported. In the review by van Alfen et al., all children showed unilateral symptoms [1]. A Pubmed search through the years after publication of this review (2000-2006) showed no reports of bilateral idiopathic neuralgic amyotrophy in children either. In adults however, it is quite common and was found in almost $30 \%$ [2]. This is the first child reported with bilateral plexus involvement. Besides, all children reported in the review by van Alfen who developed idiopathic neuralgic amyotrophy below the age of 8 weeks suffered from an associated osteomyelitis of the shoulder or arm [1]. The boy we report did not show any signs of osteomyelitis on repeated scintigraphy. His sudden paretic symptoms were preceded by a nonspecific immunologic event, most likely of viral origin, which also induced an elevation of the liver enzyme GGTP. This finding is quite interesting since Alfen et al. [2] have identified in their study a subgroup of adults who had grossly elevated liver enzymes with often an extensive bilateral brachial plexus and phrenic nerve involvement. They suggest that this subtype of neuralgic amyotrophy might be triggered by a specific antecedent infection that also generates a transient hepatitis.

\section{References}

1 van Alfen N, Schuuring J, van Engelen BGM, Rotteveel JJ, Gabreëls FJM. Idiopathic neuralgic amyotrophy in children. A distinct phenotype compared to the adult form. Neuropediatrics 2000; 31: 328-332

2 van Alfen N, van Engelen BGM. The clinical spectrum of neuralgic amyotrophy in 246 cases. Brain 2006; 129: 438-450 